EPJ Web of Conferences 95,01001 (2015)

DOI: $10.1051 /$ epjconf/20159501001

C) Owned by the authors, published by EDP Sciences, 2015

\title{
The experiment PANDA: physics with antiprotons at FAIR
}

\author{
Gianluigi Boca, on the behalf of the PANDA collaboration ${ }^{1, a}$ \\ ${ }^{1}$ Dipartimento di Fisica di Pavia and INFN, via Bassi 6, 27100 Pavia
}

\begin{abstract}
PANDA is an experiment that will run at the future facility FAIR, Darmstadt, Germany. A high intensity and cooled antiproton beam will collide on a fixed hydrogen or nuclear target covering center-of-mass energies between 2.2 and $5.5 \mathrm{GeV}$. PANDA addresses various physics aspects from the low energy non-perturbative region towards the perturbative regime of QCD. With the impressive theoretical developments in this field, e.g. lattice QCD, the predictions are becoming more accurate in the course of time. The data harvest with PANDA will, therefore, be an ideal test bench with the aim to provide a deeper understanding of hadronic phenomena such as confinement and the generation of hadron masses. A variety of physics topics will be covered with PANDA, for example: the formation or production of exotic non-qqbar charm meson states connected to the recently observed XYZ spectrum; the study of gluon-rich matter, such as glueballs and hybrids; the spectroscopy of the excited states of strange and charm baryons, their production cross section and their spin correlations; the behaviour of hadrons in nuclear matter; the hypernuclear physics; the electromagnetic proton form factors in the timelike region.

The PANDA experiment is designed to achieve the above mentioned physics goals with a setup with the following characteristics: an almost full solid angle acceptance; excellent tracking capabilities with high resolution $(1-2 \%$ at $1 \mathrm{GeV} / \mathrm{c}$ in the central region); secondary vertex detection with resolution $\approx 100$ microns or better; electromagnetic calorimetry for detections of gammas and electrons up to $10 \mathrm{GeV}$; good particle identification of charge tracks (electrons, muons, pions, kaons, protons); a dedicated interchangeable central apparatus for the hypernuclear physics; detector and data acquisition system capable of working at $20 \mathrm{MHz}$ interaction rate with an intelligent software trigger that can provide maximum flexibility.
\end{abstract}

\section{Introduction}

PANDA is an experiment that will run at the FAIR facility in Darmstadt (Frankfurt, Germany) around 2020. It will continue and extend the successful physic program started at Cern with LEAR and Fermilab with E760/E835. Presently the PANDA collaboration is composed by a group of more than 500 physicists from 56 institutions of 16 countries. The experiment will use a very high intensity antiproton beam with momentum ranging from $1.5 \mathrm{GeV} / c$ to $15 \mathrm{GeV} / c$, on a fixed proton target (pellet target or jet target). The range of energy in the center of mass covered goes from $\sqrt{s}=2.2$ up to $\sqrt{s}=5.5$ enabling the study a wide physics topic range, some of them described in the following

\footnotetext{
ae-mail: gianluigi.boca@pv.infn.it
} 
sections. The $\bar{p}$ beam will be accumulated in the HESR storage ring in two modes : a high intensity mode, with a beam current of $8.4 \mathrm{~mA}$ and stochastic cooling leading to a luminosity of $2 \times 10^{32} \mathrm{~cm}^{-2} \mathrm{~s}^{-1}$ and $\delta p / p=10^{-4}$, and a high resolution mode with electron cooling, a current of $0.84 \mathrm{~mA}$ a luminosity of $2 \times 10^{31} \mathrm{~cm}^{-2} \mathrm{~s}^{-1}$ and $\delta p / p=10^{-5}$.

\section{Some of the Panda Physics goals}

For lack of space it is impossible here to describe all physics measurements feasible in PANDA. Only a few are briefly described in the following. For a more detailed description see the PANDA Physics Book[1].

\subsection{Charmonium physics}

The $p \bar{p}$ system can form (non-exotic) states with any $\mathrm{J}^{\mathrm{PC}}$ not just $1^{--}$as in the $e^{+} e^{-}$experiments. Consequently all the charmonium states predicted by the potential models can be studied in PANDA. The mass and width resolution of the states formed is driven essentially by the resolution of the $\bar{p}$ momentum and less by the detector performances, as demonstrated by the experience of the experiment E835 at Fermilab.

Thanks to the $2 \times 10^{32} \mathrm{~cm}^{-2} \mathrm{~s}^{-1}$ luminosity, PANDA will accumulate $8 p b^{-1}$ integrated luminosity per day (assuming overall $50 \%$ efficiency) and $10^{4} \div 10^{7} c \bar{c}$ states/day. In 6 months data taking $1.5 \mathrm{fb}^{-1}$ will be accumulated, approximately 10 times better than the Fermilab experiments E760/E835 with a better detector, with better angular coverage, with magnetic field and the ability of detecting hadronic decay modes.

The charmonium states can be divided in 'conventional' $c \bar{c}$ states predicted by the quark model and 'unconventional' states whose interpretation is still far from obvious. Concerning the physics of the charmonium 'unconventional' states, PANDA will be able to study many of them in great detail; for instance the X(3872). This state was discovered by Belle in 2003[6] in the decay $X(3872) \rightarrow J / \psi \pi^{+} \pi^{-}$ and confirmed later by CDF, D0 and BaBar. Several hypothesis have been suggested to explain this narrow state : excited charmonium $\left(1^{3} D_{2}\right.$ or $\left.1^{3} D_{3}\right)$, a $D^{0} D^{* 0}$ molecule, $c \bar{c} g$ hybrid.

The $\mathrm{X}(3872)$ state is not the only puzzle in the mass spectrum. PANDA can study many of the reported states[7] : the $X^{ \pm}(3900), X(3940), X^{ \pm}(4020), X^{ \pm}(4050), X(4160), X^{ \pm}(4250), X(4260), X(4360)$, $\mathrm{X}^{ \pm}(4430), \mathrm{X}(4660)$.

The charged states have a particular importance since they are the first unambiguos sign of non- $q \bar{q}$ states.

\subsection{Gluonic excitations (hybrids, glueballs) and other exotics}

QCD allows for a state spectrum richer than the one predicted by the quark model. Gluons can be principal components of new hadrons : glueballs and hybrids. The additional gluons make possible exotic $J^{P C}$ forbidden to SU3 quark model hadrons. That is a powerful experimental signature for their discovery. Their properties are determined by the long distance features of QCD and for this reason they are very interesting. For many of these states the latest LQCD calculations improved the precision of the predictions on the mass and the width. LQCD predicts about 15 glueball states with mass accessible to PANDA (for instance the first $2^{+-}$state is predicted at $4.3 \mathrm{GeV} / c^{2}$ ). Some of them have exotic quantum numbers (oddballs) and a width of $\sim 100 \mathrm{MeV} / c^{2}$. Glueballs decay color blindly in $u \bar{u}, d \bar{d}, s \bar{s}$ and $c \bar{c}$ and can mix with normal hadronic resonances in the same mass range. Oddballs, due to their exotic $J^{P C}$ are predicted to be narrower and easier to discover in partial wave analysis. 
The glueball decays most favourable to PANDA are those into $\phi \phi$ or $\phi \eta$ if their mass is $<3.6 \mathrm{GeV} / c^{2}$ or into $J / \psi \eta$ or $J / \psi \phi$ if it is above. PANDA can form glueballs in the $p \bar{p} \rightarrow \phi \phi$ channel, with statistics two orders of magnitude better than JETSET, or $p \bar{p} \rightarrow \omega \omega, \rho \rho, K \bar{K}^{*}$. The $\eta(1475) \rightarrow K \bar{K} \pi$ state published by the OBELIX collaboration[8] can be studied in detail.

As far as the hybrids are concerned, non-charmonium candidates are the $\pi_{1}(1400)$ decaying into $\eta \pi, \rho \pi$, the $\pi_{1}(1600)$ decaying into $\eta^{\prime} \pi, \rho \pi, b_{1} \pi, f_{1} \pi, \omega \pi \pi$ the $\pi_{1}(2000)$ decaying in $f_{1} \pi, \omega \pi \pi$ and the $h_{2}(1950)$ decaying in $\omega \pi \pi$. PANDA will have the best chance to detected the $c \bar{c} g$ states with exotic quantum numbers since they are predicted to be relatively narrow and to have mass in the region above $3.5 \mathrm{GeV} / \mathrm{c}^{2}$ where the spectrum is not so populated by other large resonances.

As far as the tetraquarks and pentaquarks is concerned, PANDA will have access to these states up to $\sim 5.5 \mathrm{GeV} / c^{2}$; the reaction $p \bar{p} \rightarrow \Theta^{+} \Theta^{-}$, if it exists, could be studied near threshold.

\subsection{Electromagnetic form factors of the proton in the time-like region}

The electromagnetic form factors in the time-like region can be studied in the $p \bar{p} \rightarrow e^{+} e^{-}$reaction. To first order QCD

$$
\frac{d \sigma}{d \cos \theta^{\star}}=\frac{\pi \alpha^{2}}{8 E P}\left[\left|G_{M}\right|^{2}\left(1+\cos ^{2} \theta^{\star}\right)+\frac{4 m_{p}^{2}}{s}\left|G_{E}\right|^{2}\left(1-\cos ^{2} \theta^{\star}\right)\right]
$$

where $E$ and $P$ are the energy and momentum of the $\bar{p}$ in the $p \bar{p}$ reaction center of mass. Presently data exist up to a maximum of $Q^{2} \equiv s \sim 15 \mathrm{GeV}^{2}$ but higher $Q^{2}$ data are crucial to check the theory predictions and to check the equality between the space-like and time-like form factors for corresponding $Q^{2}$. Only the experiments E760 and E835 measured the form factors up to $15 \mathrm{GeV}^{2}$ but, due to low statistics, they had to assume $\left|G_{E}\right|=\left|G_{M}\right|$. In PANDA it will be possible to measure them without assumptions, up to $Q^{2}=29 \mathrm{GeV}^{2}$, with much wider angular acceptance and higher statistics.

\subsection{The hypernuclear physics program}

Hypernuclei are a unique possibility to study basic properties of the potential between nucleons and hyperons in a bound state. In recent years theoretical developments could predict the energy levels of the double strangeness hypernuclei. In PANDA the $\Xi$ production will be used to form double $\Lambda$ hypernuclei. In PANDA the reaction $\bar{p} p \rightarrow \Xi^{-} \Xi^{+}$and $\bar{p} n \rightarrow \Xi^{-} \Xi^{0}$ followed by rescattering of the $\Xi^{-}$ in a secondary target can be used. After stopping the $\Xi^{-}$in an external secondary target the formed $\Xi$ hypernucleus will convert in a double $\Lambda$ hypernucleus. To produce and detect such a process the PANDA central microstrip detector will be replaced by the carbon primary target followed by a small secondary target, a sandwich of silicon detectors and ${ }^{9} \mathrm{Be}$ or ${ }^{10,11} \mathrm{~B}$ or ${ }^{12,13} \mathrm{C}$ absorbers to slow down and stop the $\Xi^{-}$and identify the decay products. An array of Germanium detectors will also be placed arond the targets in order to detect the $\gamma$ produced by the de-excitation of the double $\Lambda$ hypernucleus. If the de-excitation occurs through pion emission, the latter will be detected by the rest of the central detector of PANDA.

\section{The PANDA detector}

In order to achieve its wide physics program, the detector must have full angular acceptance and good angular resolution both for charged particles and for $\gamma$. The particle identification $(\pi, K, e$ and $\mu$ ) should be excellent up to a momentum of $\sim 8 \mathrm{GeV} / c$. High momentum resolution for a relatively wide 


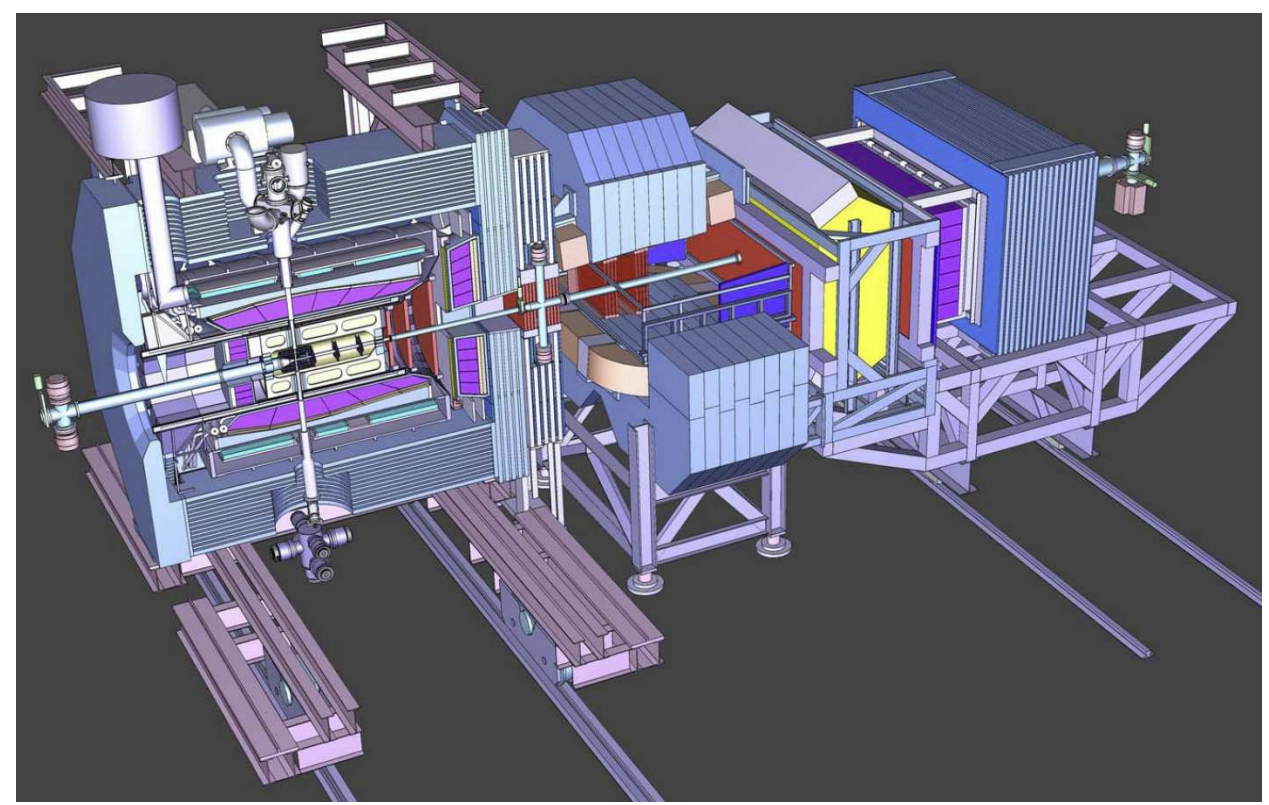

Figure 1. Top view of the PANDA apparatus

momentum range is required. The detector has to work at a very high rate $\left(2 \times 10^{7} \mathrm{~Hz}\right)$ in order to achieve the desired luminosity.

A top view of the detector is shown in fig. 1. There are two magnets. A solenoid with $2 \mathrm{~T}$ field for the central region, a dipole with an integrated field of 2 Tesla-meter in the forward region. The $\bar{p}$ beam has a moment ranging from 1.5 up to $15 \mathrm{GeV} / c$ and collides on a fixed target, a pellet target (the option of a gas jet target is also being considered). A microvertex detector made of pixel sensors and microstrip sensors with a barrel geometry surrounds the interaction region. The central detector is completed by a inner tracking detector (straw tubes), a DIRC for particle identification and an electromagnetic calorimeter. An inner time of flight detector (SciTil) made of by scintillator tiles is placed around the DIRC. Outside and around the solenoid a system of scintillator counters and drift chambers for muon detection will be placed. In the forward direction, for polar angles less than $22^{0}$, the dipole and 8 stations of multiwire drift chambers will help determining the track momentum. Particle identification will be ensured by a forward DIRC and a RICH and by time of flight counters scintillator walls. An electromagnetic calorimeter and hadron calorimeter will detect neutral particles like $\gamma$ and neutrons. In the following sections the most relevant parts of the detector are described.

\subsection{The pellet target}

Presently the preferred solution is a pellet target like the one used in the CELSIUS/WASA facility. The desired density of the frozen droplets of hydrogen in order to achieve the PANDA luminosity requirements is $3.8 \times 10^{15}$ atoms $/ \mathrm{cm}^{2}$ and presently the CELSIUS/WASA facility is very close to this goal : $2.8 \times 10^{15}$ atoms $/ \mathrm{cm}^{2}$. The pellets are blown into the vacuum beam pipe through a tube which should be as small as possible. In the present design its inner diameter is $2 \mathrm{~cm}$. 


\subsection{The microvertex detector}

The microvertex detector[9] is placed around the beam pipe and it has a barrel geometry with 5 layers. The first 3 layers are pixels $100 \times 100 \mu \mathrm{m}^{2}$. In order to reduce the material the 2 outer layers are double sided strips. There are also 5 forward wheels. The first 3 are pixels and the last two are double sided strips. In total $\sim 12$ million pixels redout channels in the barrel and forward region and $\sim 200000$ strip readout channels. The pixel technology is the same used for LHC. The pixel total thickness (including the frontend) is $\sim 500 \mu \mathrm{m}$. The digitization is performed locally with the time over threshold method, in an ASIC using the $0.13 \mu \mathrm{m}$ technology. The resolution forseen with this detector is $100 \mu \mathrm{m}$.

\subsection{The central tracker}

The Central Tracker[10] consists of a Straw Tube system, used as tracker and for $\frac{d E}{d x}$ measurement. The straw tube system will be made of layers (from 20 up to 31 layers) of drift tubes $150 \mathrm{~cm}$ long, placed in hexagonal symmetry. They fill the cylindrical zone of inner radius $15 \mathrm{~cm}$ and outer radius $42 \mathrm{~cm}$. The first 16 and last layers are aligned parallel to the beam axis, while the 16 central layers are arranged at skew angles of $3^{0}$ enabling the measurement of the $\mathrm{z}$ position of the hit with better than 1 $\mathrm{cm}$ precision. Adiacent layers are staggered with respect to each other to help resolving the left-right ambiguity. The straw diameter is $10 \mathrm{~mm}$; the gold-plated tungsten-rhenium wire diameter is $27 \mu \mathrm{m}$ and the wall thickness of the aluminized Mylar is $24 \mu \mathrm{m}$. The total number of straws is $\sim 5300$. The gas mixture is $\mathrm{Ar}-\mathrm{CO}_{2}$ with gain $\sim 10^{5}$. The $\mathrm{x}$ and $\mathrm{y}$ resolution on the single hit is $\leq 100 \mu \mathrm{m}$, while the $\mathrm{z}$ resolution is better than $0.4 \mathrm{~cm}$; the typical momentum resolution of the straw system together with the microvertex system is $1.5 \%$ at $1 \mathrm{GeV} / \mathrm{c}$ total momentum.

\subsection{The forward GEM detector}

Particles emitted at angles below $22^{\circ}$ not covered fully by the Straw Tube system will be tracked by three stations of GEM detectors placed $1.1 \mathrm{~m}, 1.4 \mathrm{~m}$ and $1.9 \mathrm{~m}$ downstream of the target. With the envisaged luminosity, the expected particle flux in the first chamber in the vicinity of the $5 \mathrm{~cm}$ diameter beam pipe is about $3 \cdot 10^{4} \mathrm{~cm}^{-2} \mathrm{~s}^{-1}$. Besides this very high flux, this detector also has to work in the $2 \mathrm{~T}$ magnetic field. Gaseous micropattern detectors based on GEM foils as amplification stages are suitable to function under these conditions. In the current layout there are three double planes with two projections per plane. The readout plane is subdivided in an outer ring with longer and an inner ring with shorter strips. The strips are arranged in two orthogonal projections per readout plane. Owing to the charge sharing between strip layers a strong correlation between the orthogonal strips can be found giving an almost $2 \mathrm{D}$ information rather than just two projections. The readout is performed by the same front-end chips as are used for the silicon microstrips. The first chamber has a diameter of $90 \mathrm{~cm}$, the last one of $150 \mathrm{~cm}$. The readout boards carrying the ASICs are placed at the outer rim of the detectors.

\subsection{Charged particle identification systems}

The charged particle identification is essential in PANDA in all the physics channels. It is achieved with a DIRC, with a RICH, by means of $\frac{d E}{d x}$, with the Time of Flight system and with the muon counters.

The central barrel DIRC is placed in the solenoid, just around the straw tube tracker, at approximately $42 \mathrm{~cm}$ radius. It is made by quartz (fused silica) bars, where the Čerenkov light is produced, arranged 
in cylindrical fashion with axis coinciding with the one of the solenoid. It measures the Čerenkov cone of particle crossing the quartz bars and from that it is possible to derive the $\beta$ of the particle. The quartz bars with $n=1.47$ will enable kaon identification starting at $460 \mathrm{MeV} / c$. The readout of the light will be achieved either with an array of 7000 photomultipliers located outside the magnetic field of the solenoid, coupled with purified water to the quartz bars, or by MicroChannelPlate PhotoMultipliers placed just outside the bars. Another option considered is the use of silicon PhotoMultipliers.

A DIRC for the forward particles at polar angles between $10^{\circ}$ and $22^{0}$ will be placed inside the solenoid, at the downstream end. The present design idea is to use a disk of fused silica as radiator, read out in $\sim 3200$ pixels. The design will ensure $\pi / K$ separation from $1 \mathrm{GeV} / c$ up to $10 \mathrm{GeV} / c$ at $\theta=0$ and up to $5 \mathrm{GeV} / c$ at $\theta=25^{\circ}$.

In the forward direction (polar angles $<10^{\circ}$ ) the particle identification will be ensured by a RICH located downstream the dipole. The radiator is made of $3^{\text {rd }}$ generation aerogel, hydrophobic, with $>80 \%$ transmittance. For the Čerenkov cone light readout a new type of multipixel hybrid GaAsP photocathode with $60 \%$ quantum efficiency in the $300-700 \mathrm{~nm}$ range is being considered. It is an avalanche diode, with 64 pixels $2 \times 2 \mathrm{~mm}^{2}$ with $<100 \mathrm{ps}$ resolution in $1.5 \mathrm{~T}$ field.

The $d E d x$ technique is used in PANDA to separate $\pi / K / p$ typically below $800 \mathrm{MeV} / c$. In the central region the straw tube system (which works in proportional mode) will measure the energy released by the charged particles. The electromagnetic and hadronic calorimeters will be able to identify $\gamma$ 's and neutrons.

Another technique exploited in PANDA is the Time of Flight measurement for charged particles. In the central region a detector called SciTil, placed around the DIRC, will serve this purpose. The present design uses $2.85 \times 2.85 \mathrm{~cm}^{2}$ tiles of fast scintillator with thickness $0.5 \mathrm{~cm}$ mechanically mounted together with the DIRC. About 5000 tiles are forseen, each read by two APDs. A time resolution of $100 \mathrm{fs}$ is expected with this detector.

A ToF scintillator wall will be placed in the forward region, behind the dipole and the forward electromagnetic calorimeter, at a distance of $\sim 7 \mathrm{~m}$ downstream the target. The wall will be $5.6 \mathrm{~m}$ wide and $1.4 \mathrm{~m}$ high and it will consist of 60 vertical scintillator strips from 5 to $10 \mathrm{~cm}$ wide. Inside the dipole 5 vertical strips, each $10 \mathrm{~cm}$ wide and $1 \mathrm{~m}$ long will be placed and read out with fibers by phototubes placed outside the dipole magnetic field. Simulations show that with the help of the tracking system a time resolution of $50 \mathrm{ps}$ can be achieved by this ToF system.

The muon identification will be achieved with a set of Iarocci proportional tubes and with scintillator counters. They will be placed outside and around the solenoid and the dipole magnets, in the inner gap of the solenoid yoke and between the hadron calorimeter planes. In that way the iron of the yoke or several planes of hadron calorimeter will act as filter for all the other particles. The angular coverage will be from $0^{0}$ up to $60^{\circ}$ in polar angle.

\subsection{The electromagnetic calorimeter}

The detection of $\gamma$ 's, with the largest possible angular coverage is crucial in PANDA. This motivates the use of four calorimeters, one inside the solenoid, in the central barrel region, covering the polar angle from $22^{0}$ to $140^{\circ}$; one in the backward end cap region of the solenoid, covering the angles from $140^{\circ}$ to almost $180^{\circ}$; one in the downstream (forward) end cap region, covering the angles from $10^{0}$ to $22^{0}$ and one in the forward region behind the ToF wall and covering the the angles $0^{0}-10^{0}$. The calorimeters must be fast, radiation hard and have excellent resolution for $\gamma$ energies from $22 \mathrm{MeV}$ to $4 \mathrm{GeV}$. The presently favoured option is the use of $\mathrm{PbWO}_{4}$ crystals $2 \mathrm{~cm} \times 2 \mathrm{~cm} \times 22 \mathrm{X}_{0}$ read out by APDs (necessary for the strong magnetic field). The barrel calorimeter has a cylindrical symmetry, 2.5 long, $0.54 \mathrm{~m}$ thick in the radial direction, and it is made of $11360 \mathrm{PbWO}_{4}$ crystals. The upstream end cap has 816 crystals, segmented in 16 slices; the forward end cap has 1 m radius with 6864 crystals. 
The expected resolution of all the first three calorimeters is $<\frac{2 \%}{\sqrt{E}}+1 \%$. The forward calorimeter is composed of Shashlyk modules of lead and scintillator sandwich read out by fibers. The expected resolution is $\frac{\sigma(E)}{E}=(1.96 \pm 0.1) \% \bigoplus \frac{(2.74 \pm 0.05) \%}{\sqrt{E}}$ with the energy in $\mathrm{GeV}$.

\subsection{The hadronic calorimeter}

The hadronic calorimeter will detect $K_{L}$ and neutrons in the forward region. It is located $8 \mathrm{~m}$ downstream the target. We plan to reuse the MIRAC calorimeter from experiment WA80 at CERN. The PANDA arrangement consists of 20+20 modules in two rows. Each module contains 100 layers composed of steel and scintillator. The total dimensions are $1.8 \mathrm{~m}$ height, $4.4 \mathrm{~m}$ wide, and $1.12 \mathrm{~m}$ long, in the beam direction, for a total of 4.8 absorption lengths. According to GEANT4 simulations it is expected to have a resolution $\frac{\sigma(E)}{E}=\frac{0.40}{\sqrt{E}}$.

\section{References}

[1] M. F. M. Lutz et al. [PANDA Collaboration], "Physics Performance Report for PANDA: Strong Interaction Studies with Antiprotons," arXiv:0903.3905 [hep-ex].

[2] S. Choi et al., Phys. Rev. Lett. 89, 102001 (2002).

[3] M. Ablikim et al. (BES III Collab.), Phys. Rev. Lett. 104, 132002 (2010).

[4] S. Dobbs et al. (CLEO Collab.), Phys. Rev. Lett. 101, 182003 (2008).

[5] M. Andreotti et al. (FNAL E835 Collab.), Phys. Rev. D72, 032001 (2005).

[6] S. Choi et al., Phys. Rev. Lett. 91, 262001 (2003).

[7] K.A. Olive et al. (Particle Data Group), Chin. Phys. C, 38, 090001 (2014).

[8] F.Nichitiu et al.(OBELIX Collab.), Phys. Lett. B545, 261 (2002).

[9] W.Erni et al. [PANDA Collaboration], "Technical Design Report for the: PANDA Micro Vertex Detector," arXiv:1207.6581 [physics.ins-det].

[10] W.Erni et al. [PANDA Collaboration], “Technical Design Report for the: PANDA Straw Tube Tracker," arXiv:1205.5441 [physics.ins-det]. 